ANL-HEP-PR-96-63

SPYTHIA, A Supersymmetric Extension of PYTHIA 5.7

\title{
S. Mrenna
}

\author{
High Energy Physics Division \\ Argonne National Laboratory \\ Argonne, IL 60439
}

\begin{abstract}
SPYTHIA is an event level Monte Carlo program which simulates particle production and decay at lepton and hadron colliders in the Minimal Supersymmetric Standard Model (MSSM). It is an extension of PYTHIA 5.7, with all of its previous capabilities. This paper is meant to supplement the PYTHIA/JETSET user manual, providing a description of the new particle spectrum, hard scattering processes, and decay modes. Several examples of using the program are provided.
\end{abstract}

\footnotetext{
${ }^{1}$ mrenna@hep.anl.gov
} 


\section{Introduction}

Recently, experiments at the Tevatron collider at Fermilab and the Large Electron Positron (LEP) collider at CERN have greatly extended our understanding of the Standard Model (SM). Several LEP energy upgrades have been staged on a migration towards LEP190, which will be replaced by the Large Hadron Collider (LHC). Prior to the operation of the LHC, the Tevatron collider will begin the Main Injector era, possibly with an extended running time, perhaps even at a higher luminosity than originally planned. Despite its phenomenological successes, the SM suffers from various theoretical problems which make it seem unlikely to be a complete theory. The rich physics program outline above is expected to definitively probe the energy regime responsible for Electroweak Symmetry Breaking (EWSB). One promising extension of the SM, which successfully addresses several of its deficiencies, is the the Minimal Supersymmetric Standard Model (MSSM) [1]. Monte Carlo simulations are useful tools for studying the phenomenological implications of a new theory, particularly for determining search strategies and optimizing detector design. SPYTHIA is an event level Monte Carlo program which simulates particle production and decay at lepton and hadron colliders in the MSSM. It is an extension of PYTHIA 5.7 [2], with all of its previous capabilities, and additional particles, hard scattering processes, and decays. Furthermore, the simulation of the MSSM Higgs sector already present in PYTHIA is extended to include the decay of Higgs bosons into MSSM particles and the decay of MSSM particles into Higgs boson.

The are already a few similar programs available. Most of the processes included in SPYTHIA are already present in the ISAJET/ISASUSY [3] event generator. In addition, a number of processes and decays relevant for lepton colliders are included in the generator SUSYGEN 《4, which is interfaced to JETSET [5] and includes initial state photon radiation. The development of SPYTHIA arose from the desire to study the phenomenology of the MSSM at lepton-lepton, lepton-hadron, and hadron-hadron colliders using the initial and final state radiation and fragmentation models of PYTHIA/JETSET. Additionally, one can perform cross checks and estimate model uncertainties with other generators.

Like the SM, the MSSM contains a number of parameters with the dimension of mass which are not fixed by the theory. Supergravity (SUGRA) inspired models reduce the number of free parameters by imposing universality and exploiting the apparent unification of gauge couplings. Five parameters fixed at the gauge coupling unification scale, $\tan \beta, M_{0}, m_{1 / 2}, A_{0}$, and $\operatorname{sign}(\mu)$, are then related to the mass parameters at the scale of EWSB by renormalization group equations [6]. ISASUSY and SUSYGEN numerically solve these equations to determine the mass parameters. Alternatively, they can input a general set of parameters. SPYTHIA operates in the second manner, with a slightly more general set of input parameters. There are three reasons for this: (1) programs already exist to calculate the SUGRA inspired mass parameters, (2) approximate analytic formulae [7] also exist which reproduce the output of ISASUSY within $\simeq 10 \%$, and (3) we desire to study a much richer phenomenology than that possible in SUGRA inspired models. The SPYTHIA input parameters are described in detail later.

In the following, it is assumed that the reader has a working knowledge of PYTHIA/JETSET? The modifications made in SPYTHIA are the sole product and responsibility of this author and not H.U. Bengtsson or T. Sjöstrand. However, these modifications will be included

${ }^{2} \mathrm{~A}$ postscript version of the user manual for PYTHIA $5.7 /$ JETSET 7.4 is available at the URL address http://thep.lu.se/tf2/staff/torbjorn/manual.ps. 
in the future release of PYTHIA 6.1. Sec. 2 explains the physics assumptions behind the implementation of supersymmetry in PYTHIA and catalogs the new particles, processes and input parameters. The parameters and routines used in the MSSM simulation are described in Sec. 3. Availability and setup of the SPYTHIA package are detailed in Sec. 4, along with some examples of its use. Conclusions are presented in Sec. 5.

\section{Simulation of Supersymmetry}

\subsection{Particle Spectrum}

SPYTHIA assumes the particle content of the MSSM. Each SM fermion has a scalar partner with the same quantum numbers. For counting purposes, the fermion states $\psi_{L}$ and $\psi_{R}$ are separate particles with scalar partners $\phi_{L}$ and $\phi_{R}$. Each SM gauge boson has a fermion partner with the same quantum numbers. The Higgs sector is extended to two complex scalar doublets, leading to 3 additional physical Higgs bosons $\mathrm{H}, \mathrm{A}$ and $\mathrm{H}^{ \pm}$to complement the SM Higgs h. Fermion higgsino partners are added for the two scalar doublets. Additionally, a light gravitino $\tilde{G}$ is included to allow studies of gauge mediated dynamical SUSY breaking [8]. The particle partners and SPYTHIA KF codes are listed in Table 1. Note that antiparticles of scalar particles are denoted by * in the text.

Because of the large Yukawa couplings and the running masses for colored particles, the interaction and mass eigenstates for the third generation sfermions can be significantly mixed. We denote the top and bottom squark mass eigenstates $\tilde{t}_{1}, \tilde{t}_{2}, \tilde{b}_{1}$, and $\tilde{b}_{2}$ to distinguish them from the nearly degenerate eigenstates for the lighter squarks. In addition, the tau slepton mass eigenstates are $\tilde{\tau}_{1}$ and $\tilde{\tau}_{2}$. The subscript 1 or 2 refers to the lightest and heaviest state respectively. In SUGRA inspired models, in the absence of mixing so that interaction eigenstates are the same as mass eigenstates, the right eigenstate is lighter than the left. In this case, for example, $\tilde{\mathrm{b}}_{1}=\tilde{\mathrm{b}}_{R}$ and $\tilde{\mathrm{b}}_{2}=\tilde{\mathrm{b}}_{L}$. For completeness, we include the sterile $\tilde{\nu}_{R}$ particles. The mixing of the partners to the electroweak gauge bosons (gauginos) and the two Higgs doublets (higgsinos) lead to the mass eigenstates for neutralino $\tilde{\mathrm{N}}_{i}$ and chargino $\tilde{\mathrm{C}}_{i}$ particles. A complete listing of the full particle spectrum, including decay channels, branching ratios, and the total width is available (as usual) through the subroutine LULIST.

\subsection{Particle Decays}

In PYTHIA, resonances are decayed so that color flow information is correctly passed from unstable particles to their decay products and no unstable colored particles are passed on to JETSET. In SPYTHIA, all Supersymmetric partners are treated as resonances. SPYTHIA assumes R-symmetry, which has two major phenomenological consequences: (1) Supersymmetric particles are produced in pairs, and (2) there is one and only one stable Supersymmetric particle. By default, the lightest superpartner (LSP) is $\tilde{\mathrm{N}}_{1}$, unless $\tilde{\mathrm{N}}_{1}$ is allowed to decay to a gravitino. In that case, $\tilde{\mathrm{N}}_{1}$ is the next to lightest superpartner (NLSP) and $\tilde{\mathrm{G}}$ is the LSP.

The decays of superpartners are calculated using the formulae of Refs. [10, 11, 12, 14]. All decays are spin averaged. For simplicity, the kinematics of three body decays of neutralinos, charginos, and the gluino are sampled using only the phase space weight. Decays involving $\tilde{b}$ and $\tilde{t}$ use the formulae of [14], so they are valid for large values of $\tan \beta$. The one loop decays $\tilde{\mathrm{N}}_{j} \rightarrow \tilde{\mathrm{N}}_{i} \gamma$ and $\tilde{\mathrm{t}} \rightarrow c \tilde{\mathrm{N}}_{1}$ are also included. 
Table 1: SPYTHIA particle KF codes.

\begin{tabular}{|c|c|c|c|c|c|}
\hline $\mathrm{KF}$ & Name & Printed & $\mathrm{KF}$ & Name & Printed \\
\hline 41 & $\tilde{\mathrm{d}}_{L}$ & d_L & 59 & $\tilde{\nu}_{\mu L}$ & numu_L \\
\hline 42 & $\tilde{\mathrm{d}}_{R}$ & $d \_R$ & 60 & $\tilde{\nu}_{\mu R}$ & numu_R \\
\hline 43 & $\tilde{\mathrm{u}}_{L}$ & u_L & 61 & $\tilde{\tau}_{1}$ & 〜au_1 \\
\hline 44 & $\tilde{\mathrm{u}}_{R}$ & u_R & 62 & $\tilde{\tau}_{2}$ & tau_2 \\
\hline 45 & $\tilde{\mathrm{s}}_{1}$ & is_L & 63 & $\tilde{\nu}_{\tau L}$ & nutau_L \\
\hline 46 & $\tilde{\mathrm{s}}_{2}$ & $s \_R$ & 64 & $\tilde{\nu}_{\tau R}$ & $\sim$ nutau_R \\
\hline 47 & $\tilde{\mathrm{c}}_{L}$ & c c_L & 65 & $\tilde{\mathrm{g}}$ & $g$ \\
\hline 48 & $\tilde{\mathrm{c}}_{R}$ & $C \_R$ & 66 & $\tilde{\mathrm{N}}_{1}$ & $\sim \mathrm{N}_{1}$ \\
\hline 49 & $\tilde{b}_{1}$ & b_1 & 67 & $\tilde{\mathrm{N}}_{2}$ & $\sim \mathrm{N}_{2}$ \\
\hline 50 & $\tilde{b}_{2}$ & b_2 & 68 & $\tilde{\mathrm{N}}_{3}$ & ${ }^{\sim} \mathrm{N}_{3}$ \\
\hline 51 & $\tilde{\mathrm{t}}_{1}$ & $\tau_{t \_1}$ & 69 & $\tilde{\mathrm{N}}_{4}$ & ${ }^{\sim} \mathrm{N}_{4}$ \\
\hline 52 & $\tilde{\mathrm{t}}_{2}$ & t_2 & 70 & $\tilde{\mathrm{C}}_{1}^{ \pm}$ & $\sim \mathrm{C}_{1}$ \\
\hline 53 & $\tilde{\mathrm{e}}_{L}$ & e_L & 71 & $\tilde{\mathrm{C}}_{2}^{ \pm}$ & ${ }^{\sim} \mathrm{C}_{2}$ \\
\hline 54 & $\tilde{\mathrm{e}}_{R}$ & $\sim e \_R$ & & & \\
\hline 55 & $\tilde{\nu}_{e L}$ & nue_L & & & \\
\hline 56 & $\tilde{\nu}_{e R}$ & nue $\_R$ & & & \\
\hline 57 & $\tilde{\mu}_{L}$ & $\mathrm{mu} \_\mathrm{L}$ & & & \\
\hline 58 & $\tilde{\mu}_{R}$ & rmu_R & 76 & $\tilde{\mathrm{G}}$ & grvtno \\
\hline
\end{tabular}

\subsection{Processes}

Tables $2-5$ are meant to update Tables $11-14$ in the Pythia 5.7 manual. The number of available processes in SPYTHIA is extended to 400, with processes 201-280 reserved for the MSSM. In processes 210 and 213, $\tilde{\ell}$ refers to both $\tilde{e}$ and $\tilde{\mu}$. For ease of readability, we have removed the subscript $L$ on $\tilde{\nu}$. $\tilde{\mathrm{t}}_{i} \tilde{\mathrm{t}}_{i}^{*}, \tilde{\tau}_{i} \tilde{\tau}_{j}^{*}$ and $\tilde{\tau}_{i} \tilde{\nu}_{\tau}^{*}$ production correctly account for sfermion mixing. Several processes are conspicuously absent from the table. For example, processes 255 and 257 would simulate the associated production of right handed squarks with charginos. Since the right handed squark only couples to the higgsino component of the chargino, the interaction strength is proportional to the quark mass, so these processes can be ignored.

Because there are so many processes involved, there are shortcuts to allow the simulation of various classes of signals. These classes are accessible through the parameter MSEL, and are listed in Table 6. 
Table 2: Subprocess codes, part 1. First column is ' + ' for processes implemented and blank for those that are only foreseen. Second is the subprocess number ISUB, and third the description of the process. The final column gives references from which the cross sections have been obtained.

\begin{tabular}{|c|c|c|c|}
\hline In & No. & Subprocess & Reference \\
\hline+ & 201 & $\mathrm{f}_{i} \overline{\mathrm{f}}_{i} \rightarrow \tilde{\mathrm{e}}_{L} \tilde{\mathrm{e}}_{L}^{*}$ & {$[13,15]$} \\
\hline+ & 202 & $\mathrm{f}_{i} \overline{\mathrm{f}}_{i} \rightarrow \tilde{\mathrm{e}}_{R} \tilde{\mathrm{e}}_{R}^{*}$ & 13,15 \\
\hline+ & 203 & $\mathrm{f}_{i} \overline{\mathrm{f}}_{i} \rightarrow \tilde{\mathrm{e}}_{L} \tilde{\mathrm{e}}_{R}^{*}+\tilde{\mathrm{e}}_{L}^{*} \tilde{\mathrm{e}}_{R}$ & 13 \\
\hline+ & 204 & $\mathrm{f}_{i} \overline{\mathrm{f}}_{i} \rightarrow \tilde{\mu}_{L} \tilde{\mu}_{L}^{*}$ & {$[13,15]$} \\
\hline+ & 205 & $\mathrm{f}_{i} \overline{\mathrm{f}}_{i} \rightarrow \tilde{\mu}_{R} \tilde{\mu}_{R}^{*}$ & 13,15 \\
\hline+ & 206 & $\mathrm{f}_{i} \overline{\mathrm{f}}_{i} \rightarrow \tilde{\mu}_{L} \tilde{\mu}_{R}^{*}+\tilde{\mu}_{L}^{*} \tilde{\mu}_{R}$ & 13 \\
\hline+ & 207 & $\mathrm{f}_{i} \overline{\mathrm{f}}_{i} \rightarrow \tilde{\tau}_{1} \tilde{\tau}_{1}^{*}$ & 13,15 \\
\hline+ & 208 & $\mathrm{f}_{i} \overline{\mathrm{f}}_{i} \rightarrow \tilde{\tau}_{2} \tilde{\tau}_{2}^{*}$ & 13,15 \\
\hline+ & 209 & $\mathrm{f}_{i} \overline{\mathrm{f}}_{i} \rightarrow \tilde{\tau}_{\sim} \tilde{\tau}_{2}^{*}+\tilde{\tau}_{1}^{*} \tilde{\tau}_{2}$ & 13 \\
\hline+ & 210 & $\mathrm{f}_{i} \overline{\mathrm{f}}_{j} \rightarrow \tilde{\ell}_{L} \tilde{\nu}_{\ell}^{*}+\tilde{\ell}_{L}^{*} \tilde{\nu}_{\ell}$ & 15 \\
\hline+ & 211 & $\mathrm{f}_{i} \overline{\mathrm{f}}_{j} \rightarrow \tilde{\tau}_{1} \tilde{\nu}_{\tau}^{*}+\tilde{\tau}_{1}^{*} \tilde{\nu}_{\tau}$ & 15 \\
\hline+ & 212 & $\mathrm{f}_{i} \overline{\mathrm{f}}_{j} \rightarrow \tilde{\tau}_{2} \tilde{\nu}_{\tau}^{*}+\tilde{\tau}_{2}^{*} \tilde{\nu}_{\tau}$ & 15 \\
\hline+ & 213 & $\mathrm{f}_{i} \overline{\mathrm{f}}_{i} \rightarrow \tilde{\nu}_{\ell}{\tilde{\nu_{\ell}}}^{*}$ & 13,, 15 \\
\hline+ & 214 & $\mathrm{f}_{i} \overline{\mathrm{f}}_{i} \rightarrow \tilde{\nu}_{\tau} \tilde{\nu}_{\tau}^{*}$ & [13, 15] \\
\hline+ & 216 & $\mathrm{f}_{i} \overline{\mathrm{f}}_{i} \rightarrow \tilde{\mathrm{N}}_{1} \tilde{\mathrm{N}}_{1}$ & 11 \\
\hline+ & 217 & $\mathrm{f}_{i} \overline{\mathrm{f}}_{i} \rightarrow \tilde{\mathrm{N}}_{2} \tilde{\mathrm{N}}_{2}$ & 11 \\
\hline+ & 218 & $\mathrm{f}_{i} \overline{\mathrm{f}}_{i} \rightarrow \tilde{\mathrm{N}}_{3} \tilde{\mathrm{N}}_{3}$ & 11 \\
\hline+ & 219 & $\mathrm{f}_{i} \overline{\mathrm{f}}_{i} \rightarrow \tilde{\mathrm{N}}_{4} \tilde{\mathrm{N}}_{4}$ & 11 \\
\hline+ & 220 & $\mathrm{f}_{i} \overline{\mathrm{f}}_{i} \rightarrow \tilde{\mathrm{N}}_{1} \tilde{\mathrm{N}}_{2}$ & 11 \\
\hline+ & 221 & $\mathrm{f}_{i} \overline{\mathrm{f}}_{i} \rightarrow \tilde{\mathrm{N}}_{1} \tilde{\mathrm{N}}_{3}$ & 11 \\
\hline+ & 222 & $\mathrm{f}_{i} \overline{\mathrm{f}}_{i} \rightarrow \tilde{\mathrm{N}}_{1} \tilde{\mathrm{N}}_{4}$ & 11 \\
\hline+ & 223 & $\mathrm{f}_{i} \overline{\mathrm{f}}_{i} \rightarrow \tilde{\mathrm{N}}_{2} \tilde{\mathrm{N}}_{3}$ & 11 \\
\hline+ & 224 & $\mathrm{f}_{i} \overline{\mathrm{f}}_{i} \rightarrow \tilde{\mathrm{N}}_{2} \tilde{\mathrm{N}}_{4}$ & 11 \\
\hline+ & 225 & $\mathrm{f}_{i} \overline{\mathrm{f}}_{i} \rightarrow \tilde{\mathrm{N}}_{3} \tilde{\mathrm{N}}_{4}$ & 11 \\
\hline+ & 226 & $\mathrm{f}_{i} \overline{\mathrm{f}}_{i} \rightarrow \tilde{\mathrm{C}}_{1}^{ \pm} \tilde{\mathrm{C}}_{1}^{\mp}$ & 12 \\
\hline+ & 227 & $\mathrm{f}_{i} \overline{\mathrm{f}}_{i} \rightarrow \tilde{\sim}_{2}^{ \pm} \tilde{\mathrm{C}}_{2}^{\mp}$ & 12 \\
\hline+ & 228 & $\mathrm{f}_{i} \overline{\mathrm{f}}_{i} \rightarrow \tilde{\mathrm{C}}_{1}^{ \pm} \tilde{\mathrm{C}}_{2}^{\mp}$ & 12 \\
\hline+ & 229 & $\mathrm{f}_{i} \overline{\mathrm{f}}_{j} \rightarrow \tilde{\mathrm{N}}_{1} \tilde{\mathrm{C}}_{\tilde{1}}^{\overline{ \pm}}$ & $11,, 12$ \\
\hline+ & 230 & $\mathrm{f}_{i} \overline{\mathrm{f}}_{j} \rightarrow \tilde{\sim}_{2}^{\tilde{\mathrm{N}}_{2}} \tilde{\sim}_{1}^{ \pm}$ & [11, 12 \\
\hline+ & 231 & $\mathrm{f}_{i} \overline{\mathrm{f}}_{j} \rightarrow \tilde{\mathrm{N}}_{3} \tilde{\mathrm{C}}_{1}^{ \pm}$ & {$[11,12$} \\
\hline+ & 232 & $\mathrm{f}_{i} \overline{\mathrm{f}}_{j} \rightarrow \tilde{\sim}_{4} \tilde{\mathrm{C}}_{\tilde{1}}^{\tilde{\mathrm{C}}_{1}^{ \pm}}$ & 11,12 \\
\hline+ & 233 & $\mathrm{f}_{i} \overline{\mathrm{f}}_{j} \rightarrow \tilde{\sim}_{1} \tilde{\mathrm{C}}_{2}^{ \pm}$ & {$[11,12$} \\
\hline+ & 234 & $\mathrm{f}_{i} \overline{\mathrm{f}}_{j} \rightarrow \tilde{\sim}_{2} \tilde{\sim}_{2}^{ \pm}$ & 111,12 \\
\hline+ & 235 & $\mathrm{f}_{i} \overline{\mathrm{f}}_{j} \rightarrow \tilde{\sim}_{3}^{\tilde{\mathrm{N}}_{3}} \tilde{\sim}_{2}^{ \pm}$ & {$[11,12]$} \\
\hline+ & 236 & $\mathrm{f}_{i} \overline{\mathrm{f}}_{j} \rightarrow \tilde{\mathrm{N}}_{4} \tilde{\mathrm{C}}_{2}^{ \pm}$ & {$[11, \overline{12}]$} \\
\hline
\end{tabular}


Table 3: Subprocess codes, part 2. First column is ' + ' for processes implemented and blank for those that are only foreseen. Second is the subprocess number ISUB, and third the description of the process. The final column gives references from which the cross sections have been obtained.

\begin{tabular}{|c|c|c|c|}
\hline In & No. & Subprocess & Reference \\
\hline+ & 237 & $\mathrm{f}_{i} \overline{\mathrm{f}}_{i} \rightarrow \tilde{\mathrm{g}} \tilde{\mathrm{N}}_{1}$ & 115 \\
\hline+ & 238 & $\mathrm{f}_{i} \overline{\mathrm{f}}_{i} \rightarrow \tilde{\mathrm{g}} \tilde{\mathrm{N}}_{2}$ & 15 \\
\hline+ & 239 & $\mathrm{f}_{i} \overline{\mathrm{f}}_{i} \rightarrow \tilde{\mathrm{g}} \tilde{\mathrm{N}}_{3}$ & 15 \\
\hline+ & 240 & $\mathrm{f}_{i} \overline{\mathrm{f}}_{i} \rightarrow \tilde{\mathrm{g}} \tilde{\mathrm{N}}_{4}$ & 15 \\
\hline+ & 241 & $\mathrm{f}_{i} \overline{\mathrm{f}}_{j} \rightarrow \tilde{\mathrm{g}} \tilde{\mathrm{C}}_{1}^{ \pm}$ & 15 \\
\hline+ & 242 & $\mathrm{f}_{i} \overline{\mathrm{f}}_{j} \rightarrow \tilde{\mathrm{g}} \tilde{\mathrm{C}}_{2}^{ \pm}$ & 15 \\
\hline+ & 243 & $\mathrm{f}_{i} \overline{\mathrm{f}}_{i} \rightarrow \tilde{\mathrm{g}} \tilde{\mathrm{g}}$ & 15 \\
\hline+ & 244 & $\operatorname{gg} \rightarrow \tilde{g} \tilde{g}$ & 15 \\
\hline+ & 246 & $\mathrm{f}_{i} \mathrm{~g} \rightarrow \tilde{\mathrm{q}}_{i L} \tilde{\mathrm{N}}_{1}$ & 15 \\
\hline+ & 247 & $\mathrm{f}_{i} \mathrm{~g} \rightarrow \tilde{\mathrm{q}}_{i R} \tilde{\mathrm{N}}_{1}$ & 15 \\
\hline+ & 248 & $\mathrm{f}_{i} \mathrm{~g} \rightarrow \tilde{\mathrm{q}}_{i L} \tilde{\mathrm{N}}_{2}$ & 15 \\
\hline+ & 249 & $\mathrm{f}_{i} \mathrm{~g} \rightarrow \tilde{\mathrm{q}}_{i R} \tilde{\mathrm{N}}_{2}$ & 15 \\
\hline+ & 250 & $\mathrm{f}_{i} \mathrm{~g} \rightarrow \tilde{\mathrm{q}}_{i L} \tilde{\mathrm{N}}_{3}$ & 15 \\
\hline+ & 251 & $\mathrm{f}_{i} \mathrm{~g} \rightarrow \tilde{\mathrm{q}}_{i R} \tilde{\mathrm{N}}_{3}$ & 15 \\
\hline+ & 252 & $\mathrm{f}_{i} \mathrm{~g} \rightarrow \tilde{\mathrm{q}}_{i L} \tilde{\mathrm{N}}_{4}$ & 15 \\
\hline+ & 253 & $\mathrm{f}_{i} \mathrm{~g} \rightarrow \tilde{\mathrm{q}}_{i R} \tilde{\mathrm{N}}_{4}$ & 15 \\
\hline+ & 254 & $\mathrm{f}_{i} \mathrm{~g} \rightarrow \tilde{\mathrm{q}}_{j L} \tilde{\mathrm{C}}_{1}^{ \pm}$ & 15 \\
\hline+ & 256 & $\mathrm{f}_{i} \mathrm{~g} \rightarrow \tilde{\mathrm{q}}_{j L} \tilde{\mathrm{C}}_{2}^{ \pm}$ & 15 \\
\hline+ & 258 & $\mathrm{f}_{i} \mathrm{~g} \rightarrow \tilde{\mathrm{q}}_{i L} \tilde{\mathrm{g}}$ & 15 \\
\hline+ & 259 & $\mathrm{f}_{i} \mathrm{~g} \rightarrow \tilde{\mathrm{q}}_{i R} \tilde{\mathrm{g}}$ & 15 \\
\hline+ & 261 & $\mathrm{f}_{i} \overline{\mathrm{f}}_{i} \rightarrow \tilde{\mathrm{t}}_{1} \tilde{\mathrm{t}}_{1}^{*}$ & 15 \\
\hline+ & 262 & $\mathrm{f}_{i} \overline{\mathrm{f}}_{i} \rightarrow \tilde{\mathrm{t}}_{2} \tilde{\mathrm{t}}_{2}^{*}$ & 15 \\
\hline+ & 263 & $\mathrm{f}_{i} \overline{\mathrm{f}}_{i} \rightarrow \underset{\sim}{\tilde{\mathrm{t}}_{1}} \tilde{\mathrm{t}}_{2}^{*}+\tilde{\mathrm{t}}_{1}^{*} \tilde{\mathrm{t}}_{2}$ & 15 \\
\hline+ & 264 & $\mathrm{gg} \rightarrow \tilde{\mathrm{t}}_{1} \tilde{\mathrm{t}}_{1}^{*}$ & 15 \\
\hline+ & 265 & $\mathrm{gg} \rightarrow \tilde{\mathrm{t}}_{2} \tilde{\mathrm{t}}_{2}^{*}$ & 15 \\
\hline+ & 271 & $\mathrm{f}_{i} \mathrm{f}_{j} \rightarrow \tilde{\mathrm{q}}_{i L} \tilde{\mathrm{q}}_{j L}$ & 15 \\
\hline+ & 272 & $\mathrm{f}_{i} \mathrm{f}_{j} \rightarrow \tilde{\mathrm{q}}_{i R} \tilde{\mathrm{q}}_{j R}$ & 15 \\
\hline+ & 273 & $\mathrm{f}_{i} \mathrm{f}_{j} \rightarrow \tilde{\mathrm{q}}_{i L} \tilde{\mathrm{q}}_{j R}+\tilde{\mathrm{q}}_{i R} \tilde{\mathrm{q}}_{j L}$ & 15 \\
\hline+ & 274 & $\mathrm{f}_{i} \overline{\mathrm{f}}_{j} \rightarrow \tilde{\mathrm{q}}_{i L} \tilde{\mathrm{q}}_{j}^{*}$ & 15 \\
\hline+ & 275 & $\mathrm{f}_{i} \overline{\mathrm{f}}_{j} \rightarrow \tilde{\mathrm{q}}_{i R} \tilde{\mathrm{q}}_{j}^{*} R$ & 15 \\
\hline+ & 276 & $\mathrm{f}_{i} \overline{\mathrm{f}}_{j} \rightarrow \tilde{\mathrm{q}}_{i L} \tilde{\mathrm{q}}_{j}^{*}+\tilde{\mathrm{q}}_{i R} \tilde{\mathrm{q}}_{j L}^{*}$ & 15 \\
\hline+ & 277 & $\mathrm{f}_{i} \overline{\mathrm{f}}_{i} \rightarrow \tilde{\mathrm{q}}_{j L} \tilde{\mathrm{q}}_{j}^{*}$ & 15 \\
\hline+ & 278 & $\mathrm{f}_{i} \overline{\mathrm{f}}_{i} \rightarrow \tilde{\mathrm{q}}_{j R} \tilde{\mathrm{q}}_{j}^{*} R$ & 15 \\
\hline+ & 279 & $\mathrm{gg} \rightarrow \tilde{\mathrm{q}}_{i L} \tilde{\mathrm{q}}_{i L}^{*}$ & 15 \\
\hline+ & 280 & $\mathrm{gg} \rightarrow \tilde{\mathrm{q}}_{i R} \tilde{\mathrm{q}}_{i}^{*} R$ & 15 \\
\hline
\end{tabular}


Table 4: Classes of processes accessible through the parameter MSEL and the individual processes codes ISUB.

\begin{tabular}{|c|l|}
\hline MSEL & Description \\
\hline 39 & All MSSM processes except Higgs production \\
40 & Squark and gluino production, ISUB $=243,244,258,259,271-280$ \\
41 & Stop pair production, ISUB $=261-265$ \\
42 & Slepton pair production, ISUB $=201-214$ \\
43 & Squark or gluino with chargino or neutralino, ISUB $=237-242,246-256$ \\
44 & Chargino-neutralino pair production, ISUB $=216-236$ \\
\hline
\end{tabular}

\section{The Parameters and Routines of the MSSM Simulation}

All of the Supersymmetric extensions of the code are included in the standard PYTHIA library. By default, Supersymmetry is not simulated. However, by setting various parameters, a rich MSSM phenomenology is available.

The parameters available to the user are stored in the FORTRAN common block COMMON/PYMSSM/IMSS $(0: 99), \operatorname{RMSS}(0: 99)$. In general, options are set by the IMSS array, while real valued parameters are set by RMSS. The entries IMSS (0) and RMSS (0) are not used, but are available for compatibility with the $\mathrm{C}$ programming language. The arrays are described below. The default values are denoted by (D).

\subsection{The MSSM Parameters}

COMMON/PYMSSM/IMSS $(0: 99), \operatorname{RMSS}(0: 99)$

Purpose: to give access to parameters that allow the simulation of the MSSM.

$\operatorname{IMSS}(1):(\mathrm{D}=0)$ level of MSSM simulation.

$=0: \quad$ No MSSM simulation.

$=1: \quad$ A general MSSM simulation. The parameters of the model are set by the array RMSS.

$=2: \quad$ An approximate SUGRA simulation using the analytic formulate of [7] to reduce the number of free parameters. In this case, only five input parameters are used. RMSS ( 1 ) is the common gaugino mass $m_{1 / 2}$, RMSS (8) is the common scalar mass $m_{0}$, RMSS (4) fixes the sign of the higgsino mass $\mu$, RMSS (16) is the common trilinear coupling $A$, and RMSS(5) is $\tan \beta=v_{2} / v_{1}$.

IMSS (2) : $(\mathrm{D}=0)$ treatment of $U(1), S U(2)$, and $S U(3)$ gaugino mass parameters.

$=0: \quad$ The gaugino parameters $M_{1}, M_{2}$ and $M_{3}$ are set by RMSS (1), RMSS (2), and RMSS (3), i.e. there is no forced relation between them.

$=1$ : The gaugino parameters are fixed by the relation $M_{1} / \alpha_{1} 3 / 5=M_{2} / \alpha_{2}=$ $M_{3} / \alpha_{3}=X$ and the parameter $\operatorname{RMSS}(1)$. If $\operatorname{IMSS}(1)=2$, then $\operatorname{RMSS}(1)$ is treated as the common gaugino mass $m_{1 / 2}$ and RMSS (20) is the GUT 
scale coupling constant $\alpha_{G U T}$, so that $X=m_{1 / 2} / \alpha_{G U T}$.

$=2: \quad M_{1}$ is set by $\operatorname{RMSS}(1), M_{2}$ by RMSS (2) and $M_{3}=M_{2} \alpha_{3} / \alpha_{2}$. In such a scenario, the $\mathrm{U}(1)$ gaugino mass behaves anomalously.

$\operatorname{IMSS}(3):(\mathrm{D}=0)$ treatment of the gluino mass parameter.

$=0$ : The gluino mass parameter $M_{3}$ is used to calculate the gluino pole mass with the formulae of [16]. The effects of squark loops can significantly shift the mass.

$=1: \quad M_{3}$ is the gluino pole mass. The effects of squark loops are assumed to have been included in this value.

$\operatorname{IMSS}(4):(\mathrm{D}=1)$ treatment of the Higgs sector.

$=0: \quad$ The Higgs sector is determined by the approximate formulae of [9] and the pseudoscalar mass $M_{A}$ set by RMSS (19).

$=1: \quad$ The Higgs sector is determined by the exact formulae of [9] and the pseudoscalar mass $M_{\mathrm{A}}$ set by RMSS(19). The pole mass for $M_{\mathrm{A}}$ is not the same as the input parameter .

$=2$ : The Higgs sector is fixed by the mixing angle $\alpha$ set by RMSS (18) and the mass values PMAS $(I, 1)$, where $I=25,35,36$, and 37 .

IMSS (7) : $(\mathrm{D}=0)$ treatment of the scalar masses in an extension of SUGRA models. The presence of additional $U(1)$ symmetries at high energy scales can modify the boundary conditions for the scalar masses at the unification scale.

$=0: \quad$ No additional $D$-terms are included. In SUGRA models, all scalars have the mass $m_{0}$ at the unification scale.

$=1: \quad \operatorname{RMSS}(23--25)$ are the values of $D_{X}, D_{Y}$ and $D_{S}$ at the unification scale in the model of [17]. The boundary conditions for the scalar masses are shifted based on their quantum numbers under the additional $U(1)$ symmetries.

IMSS (8) : $(\mathrm{D}=1)$ treatment of the $\tilde{\tau}$ mass eigenstates.

$=0$ : The $\tilde{\tau}$ mass eigenstates are calculated using the parameters RMSS $(13,14,17)$.

$=1: \quad$ The $\tilde{\tau}$ mass eigenstates are identical to the interaction eigenstates, so they are treated identically to ẽ and $\tilde{\mu}$.

IMSS (9) : $(\mathrm{D}=0)$ treatment of the right handed squark mass eigenstates for the first two generations.

$=0: \quad$ The $\tilde{\mathrm{q}}_{R}$ masses are fixed by RMSS (9). $\tilde{\mathrm{d}}_{R}$ and $\tilde{\mathrm{u}}_{R}$ are identical except for Electroweak $D$-term contributions.

= 1 : The masses of $\tilde{\mathrm{d}}_{R}$ and $\tilde{\mathrm{u}}_{R}$ are fixed by RMSS (9) and RMSS (22) respectively. $\operatorname{IMSS}(10):(\mathrm{D}=0)$ allowed decays for $\tilde{\mathrm{N}}_{2}$.

$=0:$ The second lightest neutralino $\tilde{\mathrm{N}}_{2}$ decays with a branching ratio calculated from the MSSM parameters.

$=1: \quad \tilde{\mathrm{N}}_{2}$ is forced to decay only to $\tilde{\mathrm{N}}_{1} \gamma$, regardless of the actual branching ratio. This can be used for detailed studies of this particular final state.

$\operatorname{IMSS}(11):(\mathrm{D}=0)$ choice of the lightest superpartner (LSP).

$=0: \quad \tilde{N}_{1}$ is the LSP.

$=1: \quad \tilde{N}_{1}$ is the next to lightest superparter (NLSP) and the gravitino is the LSP. The gravitino decay length is calculated from the gravitino mass set by $\operatorname{RMSS}(21)$ and the $\tilde{\mathrm{N}}_{1}$ mass and mixings.

$\operatorname{RMSS}(1)$ : If $\operatorname{IMSS}(1)=1 M_{1}$, the $\mathrm{U}(1)$ gaugino mass. If $\operatorname{IMSS}(1)=2$, then the common gaugino mass $m_{1 / 2}$. 
RMSS (2) : $M_{2}$, the $\mathrm{SU}(2)$ gaugino mass.

RMSS (3) : $M_{3}$, the $\mathrm{SU}(3)$ (gluino) mass parameter.

$\operatorname{RMSS}(4): \mu$, the higgsino mass parameter. If $\operatorname{IMSS}(1)=2$, only the sign of $\mu$ is used.

$\operatorname{RMSS}(5): \tan \beta$, the ratio of Higgs expectation values.

RMSS (6) : Left slepton mass $M_{\tilde{\ell}_{L}}$. The sneutrino mass is fixed by a sum rule.

RMSS (7) : Right slepton mass $M_{\tilde{\ell}_{R}}$.

RMSS (8) : Left squark mass $M_{\tilde{\mathrm{q}}_{L}}$. If $\operatorname{IMSS}(1)=2$, the common scalar mass $m_{0}$.

$\operatorname{RMSS}(9)$ : Right squark mass $M_{\tilde{\mathrm{q}}_{R}} \cdot M_{\tilde{\mathrm{q}}_{R}}$ when $\operatorname{IMSS}(9)=1$.

RMSS (10) : Left squark mass for the third generation $M_{\tilde{\mathrm{q}} L}$.

$\operatorname{RMSS}(11):$ Right sbottom mass $M_{\tilde{\mathrm{b}}_{R}}$.

RMSS (12) : Right stop mass $M_{\tilde{\mathrm{t}}_{R}}$ If negative, then it is assumed that $M_{\tilde{\mathrm{t}}_{R}}^{2}<0$.

RMSS (13) : Left stau mass $M_{\tilde{\tau}_{L}}$.

$\operatorname{RMSS}(14):$ Right stau mass $M_{\tilde{\tau}_{R}}$.

RMSS (15) : Bottom trilinear coupling $A_{\mathrm{b}}$.

RMSS (16) : Top trilinear coupling $A_{\mathrm{t}}$. If IMSS (1)=2, the common trilinear coupling $A$.

RMSS (17) : Tau trilinear coupling $A_{\tau}$.

RMSS (18) : Higgs mixing angle $\alpha$. This is only used when all of the Higgs parameters are set by the user, i.e IMSS (4) $=2$.

RMSS (19) : Pseudoscalar Higgs mass parameter $M_{\mathrm{A}}$.

$\operatorname{RMSS}(20):(\mathrm{D}=.041)$ GUT scale coupling constant $\alpha_{G U T}$.

$\operatorname{RMSS}(21):(\mathrm{D}=1.0)$ The gravitino mass $(\mathrm{eV})$.

$\operatorname{RMSS}(22): \tilde{\mathrm{u}}_{R}$ mass when $\operatorname{IMSS}(9)=1$.

$\operatorname{RMSS}(23): D_{X}$ contribution to scalar masses when $\operatorname{IMSS}(7)=1\left(\mathrm{GeV}^{2}\right)$.

RMSS (24) : $D_{Y}$ contribution to scalar masses when IMSS (7) $=1\left(\mathrm{GeV}^{2}\right)$.

RMSS (25) : $D_{S}$ contribution to scalar masses when $\operatorname{IMSS}(7)=1\left(\mathrm{GeV}^{2}\right)$.

COMMON/PYSSMT/ZMIX $(4,4), \operatorname{UMIX}(2,2), \operatorname{VMIX}(2,2), \operatorname{SMZ}(4), \operatorname{SMW}(2), \operatorname{SFMIX}(16,4)$

Purpose: to provide information on the neutralino, chargino, and sfermion mixing parameters. The variables should not be changed by the user.

ZMIX $(4,4)$ : the neutralino mixing matrix in the Bino-neutral Wino-Up higgsino-Down higgsino basis.

$\operatorname{UMIX}(2,2)$ : the chargino mixing matrix in the charged Wino-charged higgsino basis.

$\operatorname{VMIX}(2,2)$ : the charged conjugate chargino mixing matrix in the wino-charged higgsino basis.

SMZ (4) : the signed masses of the neutralinos.

SMW (2) : the signed masses of the charginos.

$\operatorname{SFMIX}(16,4)$ : the sfermion mixing matrices $\mathbf{T}$ in the $\mathrm{L}-\mathrm{R}$ basis, identified by the corresponding fermion, i.e. SFMIX $(6, I)$ is the stop mixing matrix. The four entries for each sfermion are $T_{11}, T_{12}, T_{21}$, and $T_{22}$.

\subsection{The MSSM Physics Routines}

The following subroutines and functions need not be accessed by the user, but are described for completeness.

SUBROUTINE PYAPPS : uses approximate analytic formulae to determine the full set of MSSM parameters from SUGRA inputs. 
SUBROUTINE PYGLUI : calculates gluino decay modes.

SUBROUTINE PYGQQB : calculates three body decays of gluinos into neutralinos or charginos and third generation fermions. These routines are valid for large values of $\tan \beta$. SUBROUTINE PYCJDC : calculates the chargino decay modes.

SUBROUTINE PYHEXT : calculates the non-Standard Model decay modes of the Higgs bosons. SUBROUTINE PYHGGM : determines the Higgs boson mass spectrum using several inputs.

SUBROUTINE PYINOM : finds the mass eigenstates and mixing matrices for the charginos and neutralinos.

SUBROUTINE PYMSIN : initializes the MSSM simulation.

SUBROUTINE PYNJDC : calculates neutralino decay modes.

SUBROUTINE PYPOLE : computes the Higgs boson masses using a renormalization group improved leading-log approximation and two loop leading-log corrections.

SUBROUTINE PYRNMT : determines the running mass of the top quark.

SUBROUTINE PYSFDC : calculates sfermion decay modes.

SUBROUTINE PYSUBH : computes the Higgs boson masses using only renormalization group improved formulae.

SUBROUTINE PYTBDY : samples the phase space for three body decays of neutralinos, charginos, and the gluino.

SUBROUTINE PYTHRG : computes the masses and mixing matrices of the third generation sfermions.

\section{Setup and Use of the SPYTHIA Generator}

The SPTYHIA code is available as a uuencoded file on the World Wide Web[3. It operates in the same fashion as the standard PYTHIA distribution. See the PYTHIA manual for a detailed example of its usage. Below, we present several examples of how to simulate MSSM phenomenology with SPYTHIA.

\subsection{Example 1: Light Stop}

The first example is an MSSM model with a light neutralino $\tilde{\mathrm{N}}_{1}$ and a light stop $\tilde{\mathrm{t}}_{1}$, so that $\mathrm{t} \rightarrow \tilde{\mathrm{t}}_{1} \tilde{\mathrm{N}}_{1}$ can occur. The input parameters are $\operatorname{IMSS}(1)=1, \operatorname{RMSS}(1)=70 ., \operatorname{RMSS}(2)=70$., $\operatorname{RMSS}(3)=225 ., \operatorname{RMSS}(4)=-40 ., \operatorname{RMSS}(5)=1.5, \operatorname{RMSS}(6)=100 ., \operatorname{RMSS}(7)=125 ., \operatorname{RMSS}(8)=250$. $\operatorname{RMSS}(9)=250 ., \operatorname{RMSS}(10)=1500 ., \operatorname{RMSS}(11)=1500 ., \operatorname{RMSS}(12)=-128 ., \operatorname{RMSS}(13)=100$. $\operatorname{RMSS}(14)=125 ., \operatorname{RMSS}(15)=800 ., \operatorname{RMSS}(16)=800 ., \operatorname{RMSS}(17)=0 .$, and $\operatorname{RMSS}(19)=400.0$. The top mass is fixed at $175 \mathrm{GeV}, \operatorname{PMAS}(6,1)=175.0$. The resulting model has $M_{\tilde{\mathrm{t}}_{1}}=55$ $\mathrm{GeV}$ and $M_{\tilde{\mathrm{N}}_{1}}=38 \mathrm{GeV}$. IMSS (1)=1 turns on the MSSM simulation. By default, there are no intrinsic relations between the gaugino masses, so $M_{1}=70 \mathrm{GeV}, M_{2}=70 \mathrm{GeV}$, and $M_{3}=225 \mathrm{GeV}$. The pole mass of the gluino is slightly higher than the parameter $M_{3}$, and the decay $\tilde{\mathrm{g}} \rightarrow \tilde{\mathrm{t}}_{1}^{*} \mathrm{t}+\tilde{\mathrm{t}}_{1} \overline{\mathrm{t}}$ occurs almost $100 \%$ of the time.

\subsection{Example 2: Approximate SUGRA}

The second example is an approximate SUGRA model. The input parameters are IMSS (1) $=2$, $\operatorname{RMSS}(1)=200 ., \operatorname{RMSS}(4)=1 ., \operatorname{RMSS}(5)=10 ., \operatorname{RMSS}(8)=800 .$, and $\operatorname{RMSS}(16)=0.0$. The resulting model has $M_{\tilde{\mathrm{d}}_{L}}=901 \mathrm{GeV}, M_{\tilde{\mathrm{u}}_{R}}=890 \mathrm{GeV}, M_{\tilde{\mathrm{t}}_{1}}=538 \mathrm{GeV}, M_{\tilde{\mathrm{e}}_{L}}=814 \mathrm{GeV}$,

\footnotetext{
${ }^{3}$ http://www.hep.anl.gov/theory/mrenna/spythia.hmtl
} 
$M_{\tilde{\mathrm{g}}}=560 \mathrm{GeV}, M_{\tilde{\mathrm{N}}_{1}}=80 \mathrm{GeV}, M_{\tilde{\mathrm{C}}_{1}^{ \pm}}=151 \mathrm{GeV}, M_{\mathrm{h}}=110 \mathrm{GeV}$, and $M_{\mathrm{A}}=883 \mathrm{GeV}$. It corresponds to the choice $M_{0}=800 \mathrm{GeV}, M_{1 / 2}=200 \mathrm{GeV}, \tan \beta=10, A_{0}=0$, and $\operatorname{sign}(\mu)>0$. The output is similar to an ISASUSY run, but there is not exact agreement.

\subsection{Example 3: ISASUSY Model}

The final example demonstrates how to convert the output of an ISASUSY run using the same SUGRA inputs into the SPYTHIA format. This requires a general model with the parameters $\operatorname{IMSS}(1)=1, \operatorname{IMSS}(3)=1, \operatorname{RMSS}(1)=83.81, \operatorname{RMSS}(2)=168.90, \operatorname{RMSS}(3)=581.83$, $\operatorname{RMSS}(4)=283.37, \operatorname{RMSS}(5)=10 ., \operatorname{RMSS}(6)=813.63, \operatorname{RMSS}(7)=804.87, \operatorname{RMSS}(8)=917.73$, $\operatorname{RMSS}(9)=909.89, \operatorname{RMSS}(10)=772.87, \operatorname{RMSS}(11)=901.52, \operatorname{RMSS}(12)=588.33, \operatorname{RMSS}(13)=813.63$, $\operatorname{RMSS}(14)=804.87, \operatorname{RMSS}(15)=610.54, \operatorname{RMSS}(16)=422.35, \operatorname{RMSS}(17)=600$, and $\operatorname{RMSS}(19)=858.412$.

\section{Conclusions}

SPYTHIA simulates the physics of the MSSM using the PYTHIA/JETSET platform. References to the underlying physics that is simulated have been provided, as well as an overview of the user interface to the program. With the appropriate choice of parameters, a wide range of MSSM models can be studied, including those with a light gravitino and extra $D$-term contributions to scalar masses.

${ }^{4}$ Information on the ISAJET and ISASUSY programs can be found on the World Wide Web at http://wwwcn1.cern.ch/asd/cernlib/mc/isajet.html 


\section{References}

[1] See, for example, H. Baer, et al., hep-ph/9503479;

H.E. Haber and G.L. Kane, Phys. Reps. 117(2-4), (1985) 75.

[2] H.U. Bengtsson and T. Sjöstrand, Computer Physics Commun. 43 (1987) 43;

T. Sjöstrand, Computer Physics Commun. 82 (1994) 74.

[3] H. Baer, F. Paige, S. Protopopescu, and X. Tata, Report No. FSU-HEP-930329.

[4] SUSYGEN documentation is available at vxcern::disk\$delphi:[katsanevas.susygen]susygen_manual.ps.

[5] M. Bengtsson and T. Sjöstrand, Computer Physics Commun. 43 (1987) 367.

[6] See, for example, D. Pierce, J.A. Bagger, K. Matchev, and R. Zhang, hep-ph/9606211.

[7] M. Drees and S.P. Martin, hep-ph/9504324.

[8] M. Dine, A.E. Nelson, Y. Nir, and Y. Shirman, Phys. Rev. D53 (1996) 2658.

[9] M. Carena, M. Quiros, and C.E.M. Wagner, Nucl. Phys. B461 (1996) 407.

[10] J. Gunion and H. Haber, Phys. Rev. D37, No. 9 (1988) 2515.

[11] A. Bartl, H. Fraas, W. Majerotto, Nucl. Phys B278 (1986) 1.

[12] A. Bartl, H. Fraas, W. Majerotto, Z. Phys. C. 30 (1986) 441.

[13] A. Bartl, H. Fraas, W. Majerotto, Z. Phys. C. 34 (1987) 411.

[14] A. Bartl, W. Majerotto, and W. Porod, Z. Phys. C. 68 (1995) 518.

[15] S. Dawson, E. Eichten and C. Quigg, Phys. Rev. D31 (1985) 1581.

[16] C. Kolda and S.P. Martin, Phys. Rev. D53 (1996) 3871.

[17] S.P. Martin and M.T. Vaughn, Phys. Rev. D50 (1994) 2282. 